\title{
Effects of Warm-Up, Post-Warm-Up, and Re-Warm-Up Strategies on Explosive Efforts in Team Sports: A Systematic Review
}

\author{
Luís Miguel Silva $^{1} \cdot$ Henrique Pereira Neiva ${ }^{1,2} \cdot$ Mário Cardoso Marques $^{1,2} \cdot$ Mikel Izquierdo $^{3}(\mathbb{0} \cdot$ \\ Daniel Almeida Marinho ${ }^{1,2}$
}

Published online: 2 July 2018

(c) Springer International Publishing AG, part of Springer Nature 2018

\begin{abstract}
Background In team sports, it is imperative that the warm-up improves acute explosive performance. However, the exact strategies, methods, and consequences of different warm-up practices remain unclear. A time delay between the warm-up and match and during half-time could negate the positive metabolic effects of the warm-up.

Objectives We conducted a systematic review to synthesize and analyze the potential effects of strategies during a warm-up (before match), post-warm-up (time between the end of warm-up and the start of a match), and re-warm-up (half-time break within a match) on explosive performance in team sports. Furthermore, we examined optimal warm-up strategies based on the included studies.

Methods We performed a search of four databases (Web of Science, Scopus, PubMed, and ScienceDirect) for original research articles published between January 1981 and August 2017. A total of 30 articles met the inclusion criteria, and the Cochrane risk of bias tool was used to assess the risk of bias. The results of the included studies were recalculated to determine effect sizes using Cohen's $d$.

Results A warm-up comprising 8 sets of 60 -m sprints $(-2.19 \%, d=1.20)$ improved sprint performance. Additionally, 7 min of dynamic exercises after 5 min of jogging improved sprint $(-7.69 \%, d=1.72)$, jumping $(8.61 \%, d=0.61)$, and agility performance $(-6.65 \%, d=1.40)$. The use of small-sided games also seems to be a valid strategy, especially for jumping performance $(6 \%, d=0.8)$. These benefits resulted from the warm-up strategies combined with some passive rest (between 2 and $10 \mathrm{~min}$ ) before the main performance. In this post-warm-up period, the use of heated garments could result in better outcomes than simple rest $(-0.89 \%, d=0.39)$. However, if the transition was longer than $15 \mathrm{~min}$, before entering the match, performing a re-warm-up with short-term explosive tasks to reactivate was the most effective approach $(-1.97 \%, d=-0.86)$. At half-time, heated garments maintained better sprint $(-1.45 \%, d=2.21)$ and jumping performance $(3.13 \%, d=1.62)$.

Conclusion Applying properly structured strategies in the warm-up and avoiding a long rest in the post-warm-up improves explosive performance. Studies tend to recommend a short active warm-up strategy (10-15 min), gradually increasing intensity ( $50-90 \%$ of maximum heart rate), and the use of heated garments soon after the warm-up to maintain muscle temperature. However, 2 min of active re-warm-up with short-term sprints and jumps should be needed for transitions longer than 15 min ( 90\% of maximum heart rate). Last, at the half-time re-warm-up, combining heated garments to maintain muscle temperature and performing an active strategy, with explosive tasks or small-sided games for 5 min before re-entering the game, resulted in better explosive performance than 15 min of resting.
\end{abstract}

Mikel Izquierdo

mikel.izquierdo@gmail.com

Extended author information available on the last page of the article 


\section{Key Points}

A shorter warm-up period of 10-15 min appears to result in better explosive performance than traditional warm-up routines currently used in team sports.

Passive strategies such as heated garments can help maintain the benefits of the warm-up during transition phases.

In transitions longer than 15 min between the end of warm-up and the start of a match or entry into a game, performing 2-min short-term explosive tasks allowed the recovery of performance levels achieved during the initial warm-up.

In commonly observed ambient conditions $\left(10-30{ }^{\circ} \mathrm{C}\right)$, at half-time, rest should be avoided, and at a minimum a heated garment that maintains muscle temperature and subsequent performance should be used.

\section{Introduction}

In training and competition, a warm-up routine has been suggested to be critical in increasing the preparedness for subsequent effort and thus maximizing performance [1]. Several preparation strategies have been investigated over the years, and recent studies have indicated that warm-up routines are generally considered to be beneficial to performance [2-4]. Data have been collected on the mechanisms related to warm-up procedures [2-5], pre-competition strategies [4-6], and re-warm-up strategies [7, 8]. Despite this information, the real-world application of warm-up strategies is still primarily based on individual experiences rather than scientific evidence $[3,4,6]$.

The warm-up is usually intended to generate a muscle temperature increase that allows several internal changes to occur, such as increased blood flow and optimized metabolic responses [2, 4]. Thus, the ideal warm-up should allow the athlete to attain an optimal muscle temperature range that limits fatigue as much as possible while maximizing performance [2,9]. Within the first 3-5 min of exercise, there is an increase in temperature that plateaus after 10-20 min of continuous exercise $[10,11]$. In commonly observed ambient conditions $\left(10-30{ }^{\circ} \mathrm{C}\right)$, body temperature declines rapidly after the warm-up, returning to baseline values after 15-20 min of passive rest $[6,10]$. With lowered body and muscle temperatures, performance tends to decline [9]. In extremely hot/cold conditions, body and muscle temperatures change differently, and it was reported that more than $1{ }^{\circ} \mathrm{C}$ of muscle temperature variation could result in impaired performance (for details on temperature and neuromuscular function, see Racinais and Oksa [9]).

In addition to the increased body temperature, studies have also reported other effects such as increased resting oxygen uptake, a post-activation potentiation (PAP) effect influenced by the previous activity of the same muscle group, enhancing muscle contractile response and thus the ability to produce power, less muscle resistance, and psychological effects $[2,4,8]$. A warm-up can be characterized as active when the participant uses physical activity, or passive when it depends on the use of external means without performance of any type of physical activity by the participant [2, 3, 12]. An active warm-up has been the most used strategy throughout the years, but passive warm-up strategies have gained relevance and have been investigated as a complement to preserve or maintain the effects during the transition time between the end of the warm-up and the start of a match, or half-time break within a match [2, 4].

Research on the use of warm-up techniques has shown benefits for performance, supporting the relevance of an active warm-up for a wide range of individual sports [13-15] and team sports [16]. However, the effectiveness of the warm-up routine appears to be dependent on many factors, such as the type of sport, athlete fitness and experience, tasks to be performed, environmental conditions, and constraints imposed by the organizers of the event $[12,17]$.

The effect of environmental temperature on performance is related to exercise duration. In both cold $\left(10^{\circ} \mathrm{C}\right)$ and hot $\left(40{ }^{\circ} \mathrm{C}\right)$ environments, power decrements during repeated sprints exercise have been observed [9]. However, this effect can be reduced, particularly in the morning when body temperature is at its lowest, by a passive warm-up [2, 9]. Studies in this scenario verified that, in a hot environment, short-term exercises of 1 and $30 \mathrm{~s}$ enhance performance, exercises of $5 \mathrm{~min}$ duration resulted in no change in physical performance, and exercises lasting $10 \mathrm{~min}$ or more were detrimental to performance [9].

Therefore, the warm-up must be designed for the specific needs of both the athlete and the sport [18]. While we found a wide range of studies examining the effect of different warm-ups on individual sports, few studies have focused on team sports, including review papers [4].

Despite the difficulties in analyzing games in a realworld context, or even simulated games, researchers should focus on team sports athletes performing relevant tasks for improving performance during game play. In team sports games, such as basketball or football, athletes must repeat short sequences of explosive efforts such as sprints, jumps, and changes of direction and speed $[19,20]$. Short-term sprints combined with short recoveries $(<60 \mathrm{~s})$ are common in sports where athletes are required to perform repeated maximal or submaximal efforts, called repeated sprint ability. This is defined as the ability to produce the best 
average performance in a series of sprints, with brief recoveries [21, 22]. Considering the vital role of repeated sprint ability, enhancing sprint performance should be a priority for athletes and sport scientists [22]. Indeed, warm-ups are essential for enhancing and attaining optimal performance in these explosive tasks [14], and can influence the results of sporting contests.

Scientific research has demonstrated the efficacy of warmup strategies for individual sports, but there is little information for those who are interested in team sports. As a result, athletes and coaches continue to design their routines based on experience and not scientific evidence. The difficulty of analyzing the effects of a warm-up in a game situation might be one of the factors impeding the focus on specific sports (for example, football). However, we suggest analyzing specific motor skills and tasks that are relevant to team sports efforts. To the best of our knowledge, no detailed systematic review has comprehensively examined the literature regarding the effects of warm-ups on team sports performance. Analyzing studies that have evaluated the effect of warm-up and re-warm-up strategies on explosive efforts would provide coaches and sports scientists with valuable knowledge and strategies to optimize programs for athletes in training or competition. Therefore, the purpose of this systematic review is to synthesize and analyze research findings on the effects of warm-up, post-warm-up (time between the end of a warm-up and the start of a match), and re-warm-up (halftime break within a match) strategies on explosive efforts in team sports. Furthermore, we recommend optimized warmup strategies for team sports performance.

\section{Methods}

\subsection{Search Strategy}

A systematic review was conducted according to PRISMA (Preferred Reporting Items for Systematic reviews and MetaAnalyses) guidelines [23]. Relevant publications published between January 1981 and August 2017 in four databases (Web of Science, Scopus, PubMed, and ScienceDirect) were identified (search results can be found in: https://doi. org/10.17504/protocols.io.pqkdmuw). The search was performed using the Boolean search method, which limited the search results with operators including AND/OR to only those documents containing relevant key terms (Table 1) in the scope of this review.

\subsection{Eligibility Criteria}

Research articles were included or excluded using criteria defined with the PICO (Population, Intervention, Comparison and Outcome) criteria [24], and the literature searches were limited to studies involving experienced team sports athletes. Theses, dissertations, and conference abstracts and proceedings were also excluded. There were no restrictions on written language, but studies were required to have an English abstract and to be published in a peer-reviewed journal. The search strategy and eligibility criteria are shown in Table 1.

\subsection{Study Selection}

The initial search identified 330 articles with potential relevance. After removing duplicates and studies that were not specific to a sports warm-up, a manual screening according to the abstract was performed, and those that were not relevant were excluded. From these studies, 90 original research

Table 1 Search strategy and inclusion/exclusion criteria based on PICO (Population, Intervention, Comparison and Outcome)

\begin{tabular}{|c|c|c|c|c|}
\hline Databases & Search terms & PICO & Inclusion criteria & Exclusion criteria \\
\hline \multirow{11}{*}{$\begin{array}{l}\text { Web of Science } \\
\text { Scopus } \\
\text { PubMed } \\
\text { ScienceDirect }\end{array}$} & \multirow{11}{*}{$\begin{array}{l}\text { Team sports } \\
\text { Warm-up } \\
\text { Re-warm-up } \\
\text { Performance } \\
\text { Prior exercise } \\
\text { Explosive effort } \\
\text { Repeated sprint } \\
\text { Vertical jump }\end{array}$} & Population & Team sports athletes & Non-team sports athletes \\
\hline & & \multirow{6}{*}{ Intervention } & Warm-up (active or passive) strategies in & \multirow{2}{*}{$\begin{array}{l}\text { No activity in control group of warm-up } \\
\text { studies }\end{array}$} \\
\hline & & & team sports & \\
\hline & & & \multirow{4}{*}{$\begin{array}{l}\text { Post-warm-up (active or passive) strategies in } \\
\text { team sports } \\
\text { Re-warm-up (active or passive) strategies in } \\
\text { team sports }\end{array}$} & \multirow{4}{*}{$\begin{array}{l}\text { Not considered by the researchers a practical } \\
\text { method to apply to team sports } \\
\text { Only included stretching routines }\end{array}$} \\
\hline & & & & \\
\hline & & & & \\
\hline & & & & \\
\hline & & \multirow[t]{3}{*}{ Comparison } & Warm-up strategies & \multirow{3}{*}{$\begin{array}{l}\text { No comparison between structured strategies } \\
\text { or control condition with pre/post-results }\end{array}$} \\
\hline & & & Post-warm-up strategies/conditions & \\
\hline & & & Re-warm-up strategies/conditions & \\
\hline & & Outcome & $\begin{array}{l}\text { Performance of explosive tasks (sprint, jump, } \\
\text { or agility tasks) }\end{array}$ & $\begin{array}{l}\text { Outcome measures not considered in review } \\
\text { Criterion test recovery in repeated tasks }>60 \mathrm{~s}\end{array}$ \\
\hline
\end{tabular}


articles were full text assessed for eligibility, and those that did not meet the inclusion criteria were excluded. For the qualitative analysis, 30 articles were included. The articles were grouped according to whether the intervention was a warm-up $(n=19)$, post-warm-up $(n=5)$, or re-warm-up $(n=6)$ strategy. A detailed flow chart describing the selection of relevant studies is shown in Fig. 1.

\subsection{Data Extraction}

From the included articles, we extracted sample size and sexes, strategies that were compared, transition times between the end of the warm-up and the criterion test, and the main results (mean and standard deviation) of sprint, jump and/or agility tasks performed. All outcome data regarding explosive tasks were grouped and analyzed individually using a Microsoft Excel 2016 spreadsheet (Microsoft, Redmond, WA, USA).

\subsection{Data Analysis}

\subsubsection{Assessment of Risk of Bias}

A bias is a systematic error, or deviation from the actual effect, in results or inferences. Two authors (LS; HN) independently assessed the risk of bias of each study against key criteria: random sequence generation, allocation concealment, blinding of participants, personnel and outcomes, incomplete outcome data, selective outcome reporting, and other sources of bias, in accordance with methods recommended by The Cochrane Collaboration [25]. The following classifications were used: low risk, high risk, or unclear risk (either lack of information or uncertainty regarding the potential for bias). The authors resolved disagreements by consensus, and a third author was consulted to resolve disagreements if necessary. Review Manager software (RevMan, The Nordic Cochrane Centre, Copenhagen, Denmark) Version 5.3.5 was used to create risk-of-bias graphs.
Fig. 1 PRISMA (Preferred Reporting Items for Systematic Reviews and Meta-Analyses) study flow diagram

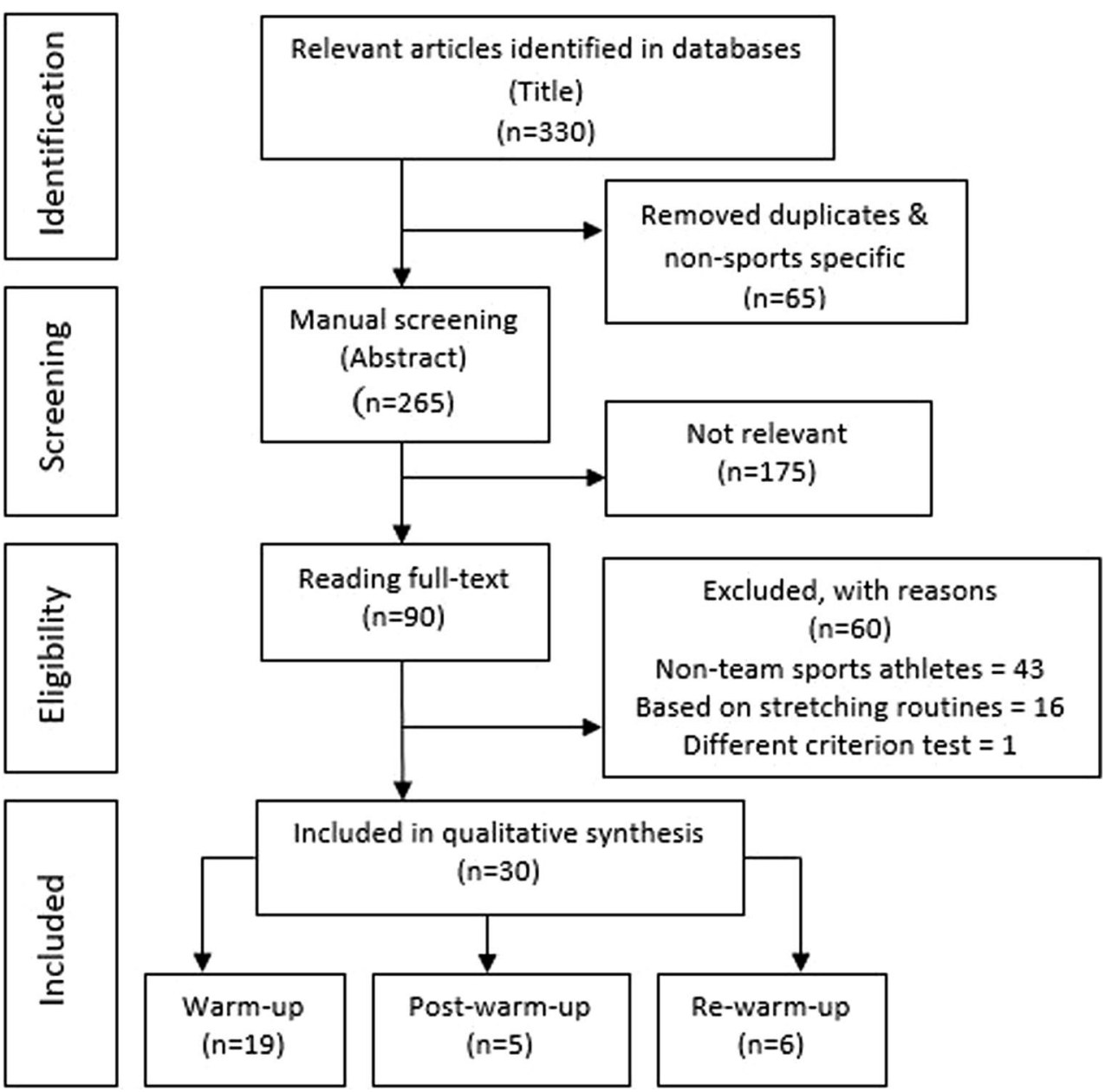




\subsubsection{Statistical Analysis}

The results of the included studies were recalculated to determine effect sizes as a measure of the difference between averages in terms of standard deviation units, which provides information about the magnitude of the observed relationship between factors [26]. Thus, this analysis was calculated using Cohen's $d$ [27], where the mean experimental value was subtracted from the mean control value and divided by the combined standard deviation. This method allowed us to determine the magnitude of differences between groups or experimental conditions for the studies that provided means and standard deviations. The magnitude of the effect was classified as small $(d=0.2)$, intermediate $(d=0.5)$, or large $(d=0.8)$ [27]. For velocity and agility outcomes, the more negative the magnitude of the result, the better the intervention compared with the control condition. For jump outcomes, the more positive the magnitude, the better the intervention. To identify the most efficient strategy, two symbols were used to match the two best strategies: ">" (better than), for the best result against the control group; and " $<$ " (worse than), for interventions that were no better than the control group.

\section{Results}

A chronological analysis of the articles that comprised this review showed a recent interest in this area of research, with almost half (47\%) of the included studies published in the last 3 years (2015-2017). Nineteen articles reported strategies for warm-up, with 60 combinations of warm-ups. Some of these studies compared the typical routines applied in team sports with different strategies. We defined this typical routine as a standard warm-up (a warm-up composed of a general aerobic warm-up, stretching routine, and specific sport exercises). In the included studies, we identified that strategies such as 8 sets of 50-m sprints $(-0.46 \%, d=-0.02)$, a 5-min jog with 5 repetition maximum (RM) leg press, or completing back squats $(4.75 \%, d=0.75)$ at the end of a typical warm-up improve explosive task performance. Among the investigations that showed a large effect size (Table 2) on sprints, performing 8 sets of 60-m sprints (60-s intervals) in the warm-up improved sprint performance $(-2.19 \%, d=1.20)$, possibly as a result of a PAP effect. This effect was observed with 5RM leg presses $(5.7 \%, d=-0.7)$. Beneficial effects were also observed on jumps after 5 min of sprinting sets at the end of the warm-up $(3.21 \%, d=1.01)$. This shows that a short specific warm-up is as effective as a long specific warm-up for sprints in football. However, 7 min of seven dynamic exercises after 5 min of jogging was associated with a general improvement in explosive tasks such as sprinting $(-7.69 \%, d=1.72)$, jumping $(8.61 \%$, $d=0.61)$, and agility performance $(-6.65 \%, d=1.40)$. Other strategies such as carrying an extra load (10\%) during all warm-up activities $(3.38 \%, d=2.79)$, participating in $12 \mathrm{~min}$ of small-sided games $(6 \%, d=0.8)$, or whole-body vibration at $50 \mathrm{~Hz}$ with a $30 \%$ extra load $(-5.12 \%, d=1.52)$ may improve performance in explosive tasks. It should be highlighted that most warm-ups were followed by some rest before the main performance (between 2 and $10 \mathrm{~min}$ ), allowing a reduction in fatigue and the participant to benefit from the potential effects of the warm-up.

Globally, applying dynamic exercises, 5RM leg presses or small-sided games seemed to favor most of the explosive efforts analyzed. In addition, the studies indicated that static stretching $(0.10 \%, d=0.03)$ was the least effective at improving sprint performance and agility.

For post-warm-up strategies, five articles verified the effectiveness of 14 conditions on the transition time after warm-up (Table 3). A progressive decrease in performance was observed in resting situations. Decreases of between $4 \%$ and $6 \%$ in sprint performance and between 12 and $20 \%$ in jump performance were observed. The use of heated garments had a moderate effect on sprint performance $(-0.89 \%$, $d=-0.39$ ). However, when this intervention was combined with three sets of five countermovement jumps with $20 \%$ body weight, a large effect was observed on sprint performance $(1.97 \%, d=0.86)$. No strategy was found to be effective in improving jump performance.

Seven studies assessed the effect of 13 re-warm-up strategies (half-time break within a match) (Table 4). A decrease in performance was identified when passive resting situations were implemented. The utilization of heated garments had the best effect size compared with resting in sprint $(-1.45 \%, d=2.21)$ and jump performance $(3.13 \%, d=1.62)$. Among all the strategies, only the use of eccentric exercise was detrimental to performance, showing the worst effect on sprint $(0.1 \% ; \pm 1.3)$ and jump performance $(-1.8 \% ; \pm 2.7)$ [28].

\subsection{Risk of Bias in the Included Articles}

Most investigations were randomized and used a crossover design (80\%). For practical reasons, most studies did not adopt a blinding design, but all made a between-group comparison. Morton [29] identified that blinding is the item that is least adhered to, as it is difficult to achieve for practical reasons (Figs. 2, 3).

\section{Discussion}

The present review aimed to analyze, from a qualitative and quantitative point of view, the findings provided in the literature regarding warm-up, post-warm-up, and rewarm-up strategies for team sports athletes. The included 
Table 2 Effects of warm-up ( $W$ ) strategies in explosive tasks

\begin{tabular}{|c|c|c|c|c|c|c|c|}
\hline \multirow[t]{2}{*}{ Reference } & \multirow[t]{2}{*}{ Subjects } & \multirow[t]{2}{*}{ Sport } & \multirow[t]{2}{*}{$W$ strategy } & \multirow{2}{*}{$\begin{array}{l}\text { Transi- } \\
\text { tion } \\
(\min )\end{array}$} & \multicolumn{3}{|c|}{ Main outcome and d } \\
\hline & & & & & Sprint & Jump & Agility \\
\hline Tillaar et al. [43] & $12 \mathrm{M}$ & Football & $\begin{array}{l}W_{1}: 10^{\prime} \text { jog }+7 \mathrm{DE}+20^{\prime} \text { jog } \\
W_{2}: w_{1}+3 \times 60 \text {-m sprints }+20^{\prime} \text { jog } \\
W_{3}: 10^{\prime} 8 \times 60 \text {-m sprints, } 60^{\prime \prime}\end{array}$ & 3 & $\begin{array}{l}W_{2}>W_{1} \\
d=-1.20 \\
-2.19 \%\end{array}$ & & \\
\hline Tillaar et al. [37] & $10 \mathrm{M}$ & Football & $\begin{array}{l}W_{1}: 19^{\prime} \text { standard } \mathrm{W} \\
W_{2}: 8 \times 50 \text {-m sprints, } 60^{\prime \prime} \text { rest }\end{array}$ & 3 & $\begin{array}{l}W_{2}>W_{1} \\
d=-0.02 \\
-0.46 \%\end{array}$ & & \\
\hline Sue et al. [35] & $9 \mathrm{M}$ & Volleyball & $\begin{array}{l}W_{1}: \text { standard } \mathrm{W} \\
W_{2}: w_{1}+8^{\prime} \mathrm{bs}+5 \mathrm{rm} \mathrm{bs}\end{array}$ & 2 & & $\begin{array}{l}W_{2}>W_{1} \\
d=0.75 \\
4.75 \%\end{array}$ & \\
\hline Barry et al. [76] & $14 \mathrm{M}$ & Rugby & $\begin{array}{l}W_{1}: 3^{\prime} \text { jog }+ \text { ds }+7 \times 10-\mathrm{m} \text { sprint } \\
W_{2}: w_{1}+5 \text { r low-load ge } \\
W_{3}: w_{1}+10 \mathrm{r} \text { low-load ge } \\
W_{4}: w_{1}+15 \mathrm{r} \text { low-load ge }\end{array}$ & 8 & $\begin{array}{l}W_{4}>W_{1} \\
d=-0.21 \\
-1.13 \%\end{array}$ & & \\
\hline Jamshidi et al. [50] & $30 \mathrm{~F}$ & Volleyball & $\begin{array}{l}W_{1}: 5^{\prime} \mathrm{jog}+\mathrm{ss}+\mathrm{de} \\
W_{2}: 4^{\prime} 26 \mathrm{~Hz} \text { vibration + squat } \\
W_{3}: w_{1}+w_{2}\end{array}$ & $\mathrm{n} / \mathrm{a}$ & $\begin{array}{l}W_{3}<W_{1} \\
d=0.01 \\
9.52 \%\end{array}$ & & $\begin{array}{l}W_{3}>W_{1} \\
d=0.00 \\
-6.05 \%\end{array}$ \\
\hline Pojskić et al. [46] & $21 \mathrm{M}$ & Football & $\begin{array}{l}W_{1}: 5^{\prime} \text { jog }+7^{\prime} \text { ds }+ \text { static squat } \\
W_{2}: w_{1}+30 \% \text { bw } \\
W_{3}: 5^{\prime} \text { jog }+7^{\prime} \text { ds }+ \text { wbv } 50 \mathrm{~Hz} \\
W_{4}: W_{3}+30 \% \text { bw }\end{array}$ & 2 & $\begin{array}{l}W_{4}>W_{1} \\
d=-1.04 \\
-3.56 \%\end{array}$ & $\begin{array}{l}W_{4}>W_{1} \\
d=0.35 \\
5.36 \%\end{array}$ & $\begin{array}{l}W_{4}>W_{1} \\
d=-1.52 \\
-5.12 \%\end{array}$ \\
\hline Pojskić et al. [49] & $21 \mathrm{M}$ & Football & $\begin{array}{l}W_{1}: 5^{\prime} \text { jog }+7^{\prime} \text { rest } \\
W_{2}: 5^{\prime} \text { jog }+ \text { ds } \\
W_{3}: 5^{\prime} \text { jog }+ \text { static half squat } \\
W_{4}: w_{3}+30 \% \text { bw }\end{array}$ & 2 & $\begin{array}{l}W_{2}>W_{1} \\
d=-1.72 \\
-7.69 \%\end{array}$ & $\begin{array}{l}W_{2}>W_{1} d=0.61 \\
8.61 \%\end{array}$ & $\begin{array}{l}W_{2}>W_{1} \\
d=-1.40 \\
-6.65 \%\end{array}$ \\
\hline Guinoubi et al. [42] & $20 \mathrm{M}$ & Football & $\begin{array}{l}W_{1}: 4^{\prime} \text { jog }+8^{\prime} \mathrm{ds}+5^{\prime} \text { sprint exer- } \\
\text { cises }+8^{\prime} \text { specific exercises } \\
W_{2}: 4^{\prime} \text { jog }+8^{\prime} \mathrm{ds}+8^{\prime} \text { specific } \\
\text { exercises }+5^{\prime} \text { sprint exercises }\end{array}$ & 10 & $\begin{array}{l}W_{2}>W_{1} \\
d=-0.78 \\
-3.47 \%\end{array}$ & $\begin{array}{l}W_{2}>W_{1} \\
d=1.01 \\
3.21 \%\end{array}$ & \\
\hline Zois et al. [16] & $10 \mathrm{M}$ & Football & $\begin{array}{l}W_{1}: 5^{\prime} \text { jog }+10^{\prime} \text { ssg ( } 3 \text { vs. } 3 \text { ) } \\
W_{2}: 5^{\prime} \text { jog }+5 \mathrm{rm} \mathrm{leg} \mathrm{press} \\
W_{3}: 23^{\prime} \text { standard } \mathrm{W}\end{array}$ & 4 & $\begin{array}{l}\text { iap: } \\
W_{2}>W_{1}>W_{3}\end{array}$ & & \\
\hline Cilli et al. [45] & $35 \mathrm{M}$ & $\begin{array}{l}\text { Basketball } \\
\text { Volleyball }\end{array}$ & $\begin{array}{l}W_{1}: \text { dynamic } w+2 \% \text { bw } \\
W_{2}: \text { dynamic } w+4 \% \text { bw } \\
W_{3} \text { : dynamic } w+6 \% \text { bw } \\
W_{4}: \text { dynamic } w+8 \% \text { bw } \\
\mathrm{W}_{5} \text { : dynamic } w+10 \% \text { bw }\end{array}$ & $\mathrm{n} / \mathrm{a}$ & & $\begin{array}{l}\mathrm{W}_{5}>W_{1} d=2.79 \\
3.38 \%\end{array}$ & \\
\hline Sander et al. [80] & $121 \mathrm{M}$ & Football & $\begin{array}{l}W_{1}: 16^{\prime} \text { standard } \mathrm{W} \\
W_{2}: w_{1}+4 \text { functional exercises }\end{array}$ & $\mathrm{n} / \mathrm{a}$ & & & $\begin{array}{l}W_{2}<W_{1} \\
d=0.32 \\
1.80 \%\end{array}$ \\
\hline Gabbett et al. [47] & $14 \mathrm{M}$ & Basketball & $\begin{array}{l}W_{1}: 7^{\prime} \text { general movements }+\mathrm{ds} \\
\text { and ss }\left(\mathrm{gw}_{1}\right)+15^{\prime} \text { open skills } \\
W_{2}: \mathrm{gw}_{1}+15^{\prime} \text { closed skill }\end{array}$ & $\mathrm{n} / \mathrm{a}$ & $\begin{array}{l}W_{2}<W_{1} \\
d=0.03 \\
1.03 \%\end{array}$ & $\begin{array}{l}W_{2}<W_{1} \\
d=-0.01 \\
-0.20 \%\end{array}$ & $\begin{array}{l}W_{2}>W_{1} \\
d=-0.09 \\
-0.45 \%\end{array}$ \\
\hline Taylor et al. [36] & $11 \mathrm{M}$ & Football & $\begin{array}{l}W_{1}: 5^{\prime} \text { jog }+ \text { specific sprints } \\
W_{2}: 5^{\prime} \text { jog }+ \text { ss }+ \text { specific sprints } \\
W_{3}: 5^{\prime} \text { jog }+ \text { ds }+ \text { specific sprints }\end{array}$ & $\mathrm{n} / \mathrm{a}$ & $\begin{array}{l}W_{3}>W_{1} \\
d=-0.03 \\
-0.13 \%\end{array}$ & & \\
\hline Okuno et al. [78] & $12 \mathrm{M}$ & Handball & $\begin{array}{l}W_{1}: 5^{\prime} \text { jog } \\
W_{2}: 5^{\prime} \text { jog }+1 \times 550 \% 1 \mathrm{rm}+1 \times 3 \\
70 \% 1 \mathrm{rm}+5 \times 190 \% 1 \mathrm{rm}\end{array}$ & $\mathrm{n} / \mathrm{a}$ & $\begin{array}{l}W_{2}>W_{1} \\
d=-0.38 \\
-1.16 \%\end{array}$ & & \\
\hline
\end{tabular}


Table 2 (continued)

\begin{tabular}{|c|c|c|c|c|c|c|c|}
\hline \multirow[t]{2}{*}{ Reference } & \multirow[t]{2}{*}{ Subjects } & \multirow[t]{2}{*}{ Sport } & \multirow{2}{*}{$W$ strategy } & \multirow{2}{*}{$\begin{array}{l}\text { Transi- } \\
\text { tion } \\
(\min )\end{array}$} & \multicolumn{3}{|c|}{ Main outcome and d } \\
\hline & & & & & Sprint & Jump & Agility \\
\hline Turki et al. [51] & $16 \mathrm{M}$ & $\begin{array}{l}\text { Football } \\
\text { Handball }\end{array}$ & $\begin{array}{l}W_{1}: 5^{\prime} \text { jog }+ \text { ads }+ \text { specific } w \\
W_{2}: 5^{\prime} \text { jog }+2 \times \text { ads }+ \text { specific } w \\
W_{3}: 5^{\prime} \text { jog }+3 \times \text { ads }+ \text { specific } w\end{array}$ & $\mathrm{n} / \mathrm{a}$ & $\begin{array}{l}W_{1} \\
d=1.17\end{array}$ & & \\
\hline Zois et al. [34] & $10 \mathrm{M}$ & Football & $\begin{array}{l}W_{1}: 5^{\prime} \text { jog }+12^{\prime} \text { ssg } 3 \text { vs. } 3 \\
W_{2}: 5^{\prime} \text { jog }+5 \text { rm leg press } \\
W_{3}: 23^{\prime} \text { standard } \mathrm{W}\end{array}$ & 4 & $\begin{array}{l}W_{2}>W_{3} \\
d=-0.7 \\
5.7 \%\end{array}$ & $\begin{array}{l}W_{1}>W_{3} \\
d=0.8 \\
6 \%\end{array}$ & $\begin{array}{l}W_{2}>W_{3} \\
d=-1.1 \\
4.7 \%\end{array}$ \\
\hline Turki et al. [44] & $20 \mathrm{M}$ & $\begin{array}{l}\text { Handball } \\
\text { Football } \\
\text { Basketball }\end{array}$ & $\begin{array}{l}W_{1}: 5^{\prime} \text { jog (control) } \\
W_{2}: 5^{\prime} \text { jog }+3 \text { rm deadlift } \\
W_{3}: 5^{\prime} \text { jog }+3 \times 3 \text { s isometric squat } \\
W_{4}: 5^{\prime} \text { jog }+3 \times 3 \text { tuck jumps } \\
\mathrm{W}_{5}: 5^{\prime} \text { jog }+3 \times \text { drop jumps } \\
\mathrm{W}_{6}: 5^{\prime} \text { jog }+ \text { ds }\end{array}$ & $\mathrm{n} / \mathrm{a}$ & & $\begin{array}{c}\mathrm{W}_{5}>W_{1} \\
d=0.67\end{array}$ & \\
\hline Rønnestad et al. [79] & $9 \mathrm{M}$ & Football & $\begin{array}{l}W_{1}: 7^{\prime} \text { jog }+3^{\prime} \text { sprints }+15 \text { squats } \\
W_{2}: w_{1}+\text { wbv } 30 \mathrm{~Hz} \\
W_{3}: w_{1}+\text { wbv } 50 \mathrm{~Hz}\end{array}$ & 1 & $\begin{array}{l}W_{3}>W_{1} \\
d=-0.20 \\
-0.72 \%\end{array}$ & & \\
\hline Beckett et al. [52] & $12 \mathrm{M}$ & $\begin{array}{l}\text { Football } \\
\text { Rugby } \\
\text { Hockey }\end{array}$ & $\begin{array}{l}W_{1}: 5^{\prime} \text { jog }+\mathrm{de}+3 \times \text { rsa } \\
W_{2}: 5^{\prime} \text { jog }+\mathrm{de}+3 \times \text { rsa }+ \text { ss } \\
W_{3}: 5^{\prime} \text { jog }+\mathrm{de}+3 \times \operatorname{cod} \\
W_{4}: 5^{\prime} \text { jog }+\mathrm{de}+3 \times \operatorname{cod}+\mathrm{ss}\end{array}$ & 4 & $\begin{array}{l}W_{2}<W_{1} \\
d=0.03 \\
0.10 \%\end{array}$ & & $\begin{array}{l}W_{4}>W_{3} \\
d=-0.09 \\
-0.49 \%\end{array}$ \\
\hline
\end{tabular}

$a d s$ active dynamic stretch, $b s$ back squat, $b w$ body weight, cod change of direction, de/s dynamic exercises/stretch, $f$ female, $g e$ gluteal exercises, iap intermittent activity protocol, $M$ male; $r$ repetition, $r m$ repetition maximum, $r s a$ repeated sprint ability, $s s$ static stretch, $s s g$ small sided game, $w b v$ whole body vibration

Table 3 Effects of post-warm-up $(P)$ strategies in explosive tasks

\begin{tabular}{|c|c|c|c|c|c|c|c|}
\hline \multirow[t]{2}{*}{ References } & \multirow[t]{2}{*}{ Subjects } & \multirow[t]{2}{*}{ Sport } & \multirow{2}{*}{$\begin{array}{l}\text { Warm-up } \\
\text { (min) }\end{array}$} & \multirow{2}{*}{$\begin{array}{l}\text { Transition } \\
\text { (min) }\end{array}$} & \multirow[t]{2}{*}{$P$ strategy } & \multicolumn{2}{|c|}{ Main outcome and $d$} \\
\hline & & & & & & Sprint & Jump \\
\hline Crowther et al. [59] & $2 \mathrm{M}$ & Basketball & 20 & 20 & $P_{1}$ : rest & & $\begin{array}{l}\text { Player 1: } \downarrow 15.4 \% \\
\text { Player 2: } \downarrow 11.9 \%\end{array}$ \\
\hline West et al. [62] & $15 \mathrm{M}$ & Rugby & 25 & 20 & $\begin{array}{l}P_{1}: \text { nta } \\
P_{2}: \text { bsg } \\
P_{3}: \text { nta }+3 \times 5 \mathrm{cmj}+20 \% \text { bw } \\
P_{4}: \text { bsg }+3 \times 5 \mathrm{cmj}+20 \% \text { bw }\end{array}$ & $\begin{array}{l}P_{4}>P_{1} \\
d=-0.86 \\
-1.97 \%\end{array}$ & $\begin{array}{l}P_{4}<P_{1} \\
d=0.00 \\
-0.02 \%\end{array}$ \\
\hline Alberti et al. [61] & $81 \mathrm{M}$ & Basketball & 15 & 20 & $\begin{array}{l}P_{1}: \text { control } \\
P_{2}: \text { sitting } \\
P_{3}: \text { standing } \\
P_{4}: \text { tapping } \\
P_{5}: 1 \mathrm{bm}\end{array}$ & & $\begin{array}{l}P_{3}<P_{1} \\
d=-0.07 \\
-0.79 \%\end{array}$ \\
\hline Kilduff et al. [63] & $20 \mathrm{M}$ & Rugby & 25 & 15 & $\begin{array}{l}P_{1}: \text { nta } \\
P_{2}: \text { bsg }\end{array}$ & $\begin{array}{l}P_{2}>P_{1} \\
d=-0.39 \\
-0.89 \%\end{array}$ & $\begin{array}{l}P_{2}<P_{1} \\
d=-0.02 \\
-0.13 \%\end{array}$ \\
\hline Galazoulas et al. [60] & $\begin{array}{l}8 \mathrm{M} \\
8 \mathrm{~F}\end{array}$ & Basketball & 25 & 40 & $\begin{array}{l}P_{1}: 10^{\prime} \text { rest } \\
P_{2}: 20^{\prime} \text { rest } \\
P_{3}: 30^{\prime} \text { rest } \\
P_{4}: 40^{\prime} \text { rest }\end{array}$ & $\downarrow 4-6 \%$ & $\downarrow 13-20 \%$ \\
\hline
\end{tabular}

$b s g$ blizzard survival garment, $b w$ body weight, $c m j$ countermovement jump, $F$ female, $l b m$ low back mobilization, $M$ male, $n t a$ normal training attire, $\downarrow$ decline in performance 
Table 4 Effects of re-warm-up (Rw) strategies in explosive tasks

\begin{tabular}{|c|c|c|c|c|c|c|}
\hline \multirow[t]{2}{*}{ Reference } & \multirow[t]{2}{*}{ Subjects } & \multirow[t]{2}{*}{ Sport } & \multirow{2}{*}{$\begin{array}{l}\text { Half-time } \\
(\mathrm{min})\end{array}$} & \multirow[t]{2}{*}{ Rw strategy } & \multicolumn{2}{|c|}{ Main outcome and d } \\
\hline & & & & & Sprint & Jump \\
\hline Abade et al. [28] & $22 \mathrm{M}$ & Football & 15 & $\begin{array}{l}\mathrm{Rw}_{1}: 12^{\prime} \text { rest (control) } \\
\mathrm{Rw}_{2}: 6^{\prime} \text { rest }+ \text { eccentric exercise } \\
\mathrm{Rw}_{3}: 6^{\prime} \text { rest }+4 \times 5 \text { jumps } \\
\mathrm{Rw}_{4}: 6^{\prime} \text { rest }+\mathrm{rcod}\end{array}$ & $\begin{array}{l}\mathrm{Rw}_{3}>\mathrm{Rw}_{1} \\
-2.9 \%\end{array}$ & $\begin{array}{l}\mathrm{Rw}_{3}>\mathrm{Rw}_{1} \\
3.8 \%\end{array}$ \\
\hline Russel et al. [68] & $18 \mathrm{M}$ & Rugby & 15 & $\begin{array}{l}\mathrm{Rw}_{1}: \text { nta } \\
\mathrm{Rw}_{2}: \text { bsg }\end{array}$ & $\begin{array}{l}\mathrm{Rw}_{2}>\mathrm{Rw}_{1} \\
d=-2.21 \\
-1.45 \%\end{array}$ & $\begin{array}{l}\mathrm{Rw}_{2}>\mathrm{Rw}_{1} \\
d=1.62 \\
3.13 \%\end{array}$ \\
\hline Edholm et al. [67] & $22 \mathrm{M}$ & Football & 15 & $\begin{array}{l}\mathrm{Rw}_{1}: \text { traditional half-time break } \\
\mathrm{Rw}_{2}: 7^{\prime} \text { rest }+7^{\prime}: \text { jog and calisthenics }\end{array}$ & $\begin{array}{l}\mathrm{Rw}_{2}>\mathrm{Rw}_{1} \\
d=-0.72 \\
-2.02 \%\end{array}$ & $\begin{array}{l}\mathrm{Rw}_{2}>\mathrm{Rw}_{1} \\
d=0.29 \\
3.02 \%\end{array}$ \\
\hline Lovell et al. [77] & $10 \mathrm{M}$ & Football & 15 & $\begin{array}{l}\mathrm{Rw}_{1}: \text { rest (control) } \\
\mathrm{Rw}_{2}: 9^{\prime} \text { rest }+5^{\prime} \text { iae } \\
\mathrm{Rw}_{3}: 9^{\prime} \text { rest }+5^{\prime} \text { wbv } 40 \mathrm{~Hz}\end{array}$ & $\mathrm{Rw}_{2}>\mathrm{Rw}_{1}$ & $\mathrm{Rw}_{2}>\mathrm{Rw}_{1}$ \\
\hline Zois et al. [66] & $8 \mathrm{M}$ & Football & 15 & $\begin{array}{l}\mathrm{Rw}_{1}: 15^{\prime} \text { rest (control) } \\
\mathrm{Rw}_{2}: 10^{\prime} \text { rest }+5 \mathrm{rm} \text { leg press } \\
\mathrm{Rw}_{3}: 8^{\prime} \text { rest }+7^{\prime} \mathrm{ssg}\end{array}$ & $\begin{array}{l}\mathrm{Rw}_{2}>\mathrm{Rw}_{1} \\
d=-0.6 \\
-4.6 \%\end{array}$ & $\begin{array}{l}\mathrm{Rw}_{2}>\mathrm{Rw}_{1} \\
d=0.4 \\
3 \%\end{array}$ \\
\hline Mohr et al. [65] & $9 \mathrm{M}$ & Football & 15 & $\begin{array}{l}\mathrm{Rw}_{1}: 15^{\prime} \text { rest (control) } \\
\mathrm{Rw}_{2}: 7^{\prime} \text { rest }+7^{\prime} \text { jog }+ \text { exercises at } 135 \text { beat } / \\
\min \end{array}$ & $\begin{array}{l}\mathrm{Rw}_{2}>\mathrm{Rw}_{1} \\
d=-0.66 \\
-0.67 \%\end{array}$ & \\
\hline
\end{tabular}

bsg blizzard survival garment, iae intermittent agility exercise, $M$ male, $n t a$ normal training attire, $r c o d$ repeated change of direction, $r m$ repetition maximum, ssg small-sided game, $w b v$ whole body vibration

studies identified substantial improvements in explosive activities (i.e., sprinting, jumping, agility) using different warm-up strategies. Although numerous studies have been conducted, to date, no consensus has been reached regarding an optimal strategy or protocol to apply in team sports. Of the 19 included articles that assessed warm-up strategies, $69 \%$ showed an improvement in sprint performance, $87.5 \%$ showed an improvement in jump performance, and $83 \%$ showed an improvement in agility performance. These improvements indicated that a properly structured strategy can substantially increase athlete performance in many different sports. However, some gaps were found between studies that make comparing the results difficult. Different control groups (jogging, standard routines, or structured strategy) and different warm-up volumes and tasks were used, making it difficult to directly compare the magnitude of the effect between strategies. Additionally, the lack of blinding and allocation concealment of the athletes might represent a potential risk of bias in the results, as the efforts may not have been natural.

Therefore, to define a properly structured warm-up strategy, it is important to know which variables are critical (type, composition, intensity, volume, and transition period) for optimizing subsequent explosive performance tasks. Nevertheless, studies tend to recommend a short active warm-up strategy that progresses in intensity until maximal effort is attained close to the end of the warm-up.
Shortly afterwards, a passive strategy should be used in the transition time before the game, and a re-warm-up should be performed before entering the game, with short-term sprints and jumps for longer transition times. Finally, resting should be avoided at half-time, and a passive strategy or an active strategy should be incorporated as a re-warm-up before reentering the game.

\subsection{Warm-Up Structure (Volume, Intensity, Tasks)}

The typical pre-match warm-up protocol used in team sports consists of a general warm-up phase of sub-maximal aerobic exercises, followed by a specific phase with stretching routines and sports-specific skill exercises [3, 30, 31]. These warm-up programs are traditionally designed to focus on energy systems and physiological processes, while the neurological aspects of the warm-up are often overlooked [18]. Moreover, the warm-up period usually lasts a long period (>20 min), which could introduce a risk of cumulative fatigue, compromising subsequent performance, as suggested in sports such as cycling and rowing [32, 33]. In three studies, athletes exhibited poorer sprint, jump, and agility performance after a standard warm-up than they did using other strategies such as 12-min small-sided games, 5RM leg press, or 8-min back squats [16, 34, 35]. In addition, two of the studies reported that a short warm-up might have the 
Fig. 2 Judgments about each risk-of-bias item for each included study. + indicates low risk, ? indicates unclear risk, - indicates high risk

same benefits on performance as a long warm-up and may be more practical $[36,37]$. Likewise, the 23-min professional warm-up applied in the study by Zois et al. [34] resulted in a 4-6\% poorer performance in explosive tasks compared with other shorter strategies (for example, 12 min of small-sided games or 5RM leg press).

These data agreed with results obtained in cycling, for example, the work of Tomaras and MacIntosh [32]. These authors reported that a warm-up performed at an intensity too high for longer than needed could result in fatigue and impair subsequent short-term all-out performance in track cyclists. This might be caused by a reduction in energy stores and a decline in heat storage capacity. However, some athletes seemed to feel psychologically more prepared after a 25-min warm-up than with a shorter warm-up, an effect that was independent of the decrease in physical performance [38]. Still, we may conclude that performing a shorter warm-up routine for between 10 and 15 min provides the same or more benefits than a longer routine, avoiding increased fatigue and leaving more free time for other tactical interventions.

Research suggests that an optimum preparation strategy should provide sufficient intensity ( $\sim 40-60 \%$ maximum rate oxygen uptake) and duration (5-10 $\mathrm{min})$, followed by a proper recovery (5 min) [12, 18, 39]. Explosive tasks such as sprints and jumps seemed to require a more strenuous warm-up intensity $\left[\sim 90 \%\right.$ of maximum heart rate $\left(\mathrm{HR}_{\max }\right)$ plus level 16 of rating of perceived exertion] and recovery to achieve high levels of explosive force production at the initial stages of a match [40, 41]. However, this differs from the usual tasks performed in real-world contexts. Three studies showed that completing some sprint tasks at the end of the warm-up led to a $2-3 \%$ improvement in sprint performance [36, 42, 43]. Similarly, for jump performance, the application of sprint tasks seems to boost performance by $3 \%$ [42] and post-activation potentiation can be induced by three sets of three tuck jumps [44].

To the best of our knowledge, no studies have specifically evaluated warm-up intensity in team sports. The only studies that focused on intensity applied different postactivation potentiation strategies by using muscle stimulation. Strategies such as a 5RM leg press [16, 34], dynamic exercise with an additional load of $10 \%$ body weight [45], or whole-body vibration $50 \mathrm{~Hz}$ plus a $30 \%$ body weight load [46] seem to improve performance. However, these studies were conditioned by the use of specific muscle strength measuring and evaluation apparatus. Therefore, based on these findings, we may conclude that structuring a shorter warm-up (10-15 $\mathrm{min})$, progressive in intensity

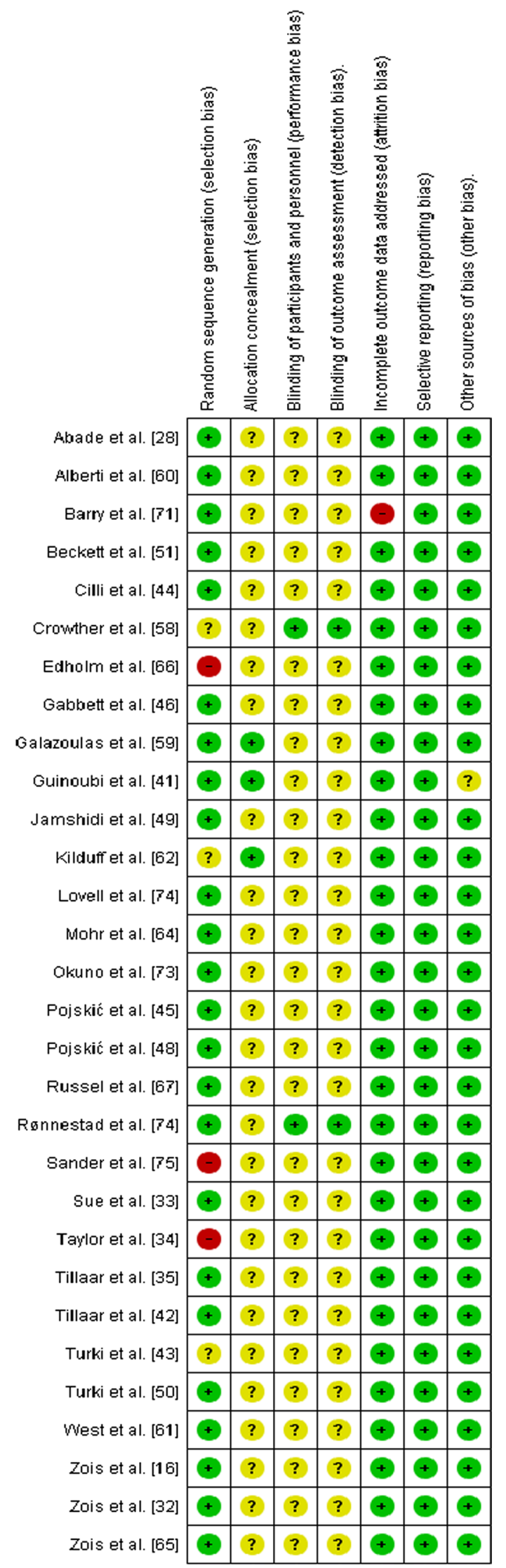


Fig. 3 Risk-of-bias item presented as percentages across all included studies
Random sequence generation (selection bias)

Allocation concealment (selection bias)

Blinding of participants and personnel (performance bias)

Blinding of outcome assessment (detection bias).

Incomplete outcome data addressed (attrition bias)

Selective reporting (reporting bias)

Other sources of bias (other bias).

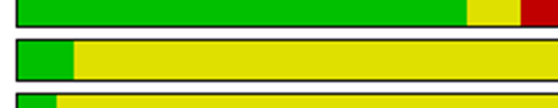

$\square$

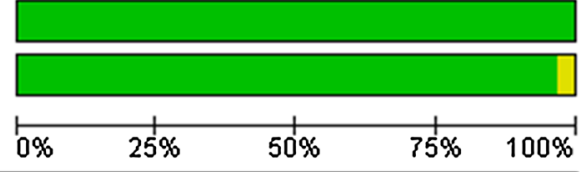

$\begin{array}{lllll}0 \% & 25 \% & 50 \% & 75 \% & 100 \%\end{array}$

Low risk of bias

Unclear risk of bias $\left(\sim 50-90 \% \mathrm{HR}_{\max }\right)$, with open or closed skills exercises [47], and ending the warm-up with sprint tasks ( 90\% $\mathrm{HR}_{\max }$ ) may be an optimal strategy. Moreover, during a team sport competition, fatigue will cause the players' performance to decrease at a certain point in time. Reducing the duration and/or intensity of the warm-up will probably delay fatigue and lead to higher overall performance during the match.

Currently, coaches worldwide typically implement stretching routines (static, dynamic, ballistic, or proprioceptive neuromuscular facilitation stretching) as an important part of their warm-up preparations, believing that they improve performance and range of motion and reduce injury risk [30]. However, there is little scientific evidence that supports the benefits of these practices. A recent study verified that a warm-up complemented with stretching exercises positively influences repeated sprint ability, especially after the first sprint [48]. Among the investigations with the best results, we found that dynamic stretching at the end of the warm-up improved explosive tasks by up to $9 \%[49,50]$. One study reported that completing one set of active dynamic stretching, and no more, may result in better sprint performance [50]. Additionally, Pojskić et al. [46] observed 6-8\% better performance in explosive tasks following dynamic stretching at the end of the warm-up, which can be a feasible option before sprint tasks [36]. Conversely, a considerable number of studies indicated that static stretching and proprioceptive neuromuscular facilitation stretching performed prior to strength, power-dominant, speed, and agility activities result in performance deficits and should normally be avoided [52-57]. However, recent findings suggested that performing dynamic movements after static stretching could have a positive effect on performance [47, 58]. Still, dynamic stretching generally can be recommended in the period immediately prior to activity, while static and proprioceptive neuromuscular facilitation stretching are probably best after activity [56]. It seems clear that an acute bout of stretching will decrease the acute ability to generate a maximal force [53], but it may be valid to apply a dynamic stretching routine in the middle of the warm-up or just before a maximum intensity exercise to potentiate performance.

\subsection{Post-Warm-Up Transition Time Effects}

Another important factor that might impair subsequent performance is the time spent by athletes in resting situations, such as between the warm-up and the match and during half-time. Usually, team sports games take approximately 12 min of transition time between the warm-up and the start of the game [4, 6], and this interval could eventually negate the positive metabolic effects of the warm-up. In addition, not all athletes begin to play shortly after the warm-up, as they may be substitutes. Accordingly, research indicated that substitutes might be physiologically underprepared when they are called upon, as just 6 min after warm-up, the core temperature significantly decreased, and heart rate and muscle temperature returned to baseline values [59]. Similarly, Galazoulas et al. [60] reported that resting resulted in rapid and highly associated decreases in temperature and performance, with the greatest impact on tasks demanding explosive and high-velocity efforts.

All the included articles identified a progressive decrease in performance with the traditional passive rest period. The reduction in jumping, sprint, and agility performance increased with resting time. After $20 \mathrm{~min}$ of rest, athletes showed a decrease of approximately $15 \%$ in jumping performance [59]. Extending the rest to $40 \mathrm{~min}$, jumping performance decreased $20 \%$ and sprint performance about $6 \%$ [60]. Additionally, one of the studies found that standing up 20 mins after the warm-up reduced the decrease in performance compared with sitting on the bench, which is more common and is significantly unfavorable to explosive tasks [61]. However, owing to restrictions imposed by the rules of some team sports games, the athlete is not allowed to stand during the game and must remain seated. Because of these constraints, the use of passive warm-up strategies for 
transitions could help maintain optimal performance, diminishing the decline in force production and sprint performance [62]. Moreover, two studies identified some strategies to mitigate possible losses in performance $[62,63]$. These authors showed that the application of heated garments after the warm-up can significantly maintain the benefits gained by the warm-up or at least reduce their decline. However, it seems even more beneficial to combine this passive strategy with an active strategy (three sets of five countermovement jumps with $20 \%$ body weight) [62]. This finding is relevant to athletes who spend more time on the bench and suggests that these athletes should combine a post-warm-up strategy with a re-warm-up before entering the game.

\subsection{Half-Time Re-Warm-Up}

Team sports are usually played with a half-time break of 10-20 min in the middle of the game, which is used to rest, rehydrate, address injuries, and receive tactical instructions [9]. However, studies have identified a decrease in physical and cognitive performance associated with the traditional rest time. All the included articles found that a passive rest period decreased performance in commonly observed ambient conditions $\left(10-30{ }^{\circ} \mathrm{C}\right)$. It should be noted that different results were found under specific conditions, such as hot environments, resulting in different muscle temperature responses [64]. Despite these conditions not being usual during matches, we should be aware that football matches performed in the heat caused muscle temperatures above optimal after the end of the first half, and perhaps influenced values during rest time and performance in the second half [64].

Knowing that temperature rapidly decreases, researchers have recently been interested in investigating strategies to prevent this decline. This could be particularly relevant in cold ambient conditions. However, studies on this topic are scarce and have mostly focused on football. Four studies on the effects of a re-warm-up found that active strategies, for example, 7-8 min of running with an intensity of $\sim 70 \% \mathrm{HR}_{\max }$ [65-67], as well as a post-activation strategy of four sets of five jumps [28], were better for subsequent performance than the traditional interval rest. However, these results should be interpreted carefully, as one study reported that eccentric exercise could increase the decline in performance and that it should be avoided [28].

In accordance with the evidence observed during postwarm-up transitions, passive strategies appear safe to use and may prevent physiological losses that cause performance impairment. However, only one study focused on this matter and reported that using heated garments during the entire half-time period had a major positive impact compared with resting alone [68]. Passive strategies and active strategies seem to be adequate to maintain or improve performance; however, none of the included studies investigated the benefits of a combination of both strategies during half-time. In a theoretical model for re-warm-up, a combination of heat maintenance strategies (active and/or passive), hormonal priming (verbal persuasion, feedback, and video clips prior to exercise), and caffeine and carbohydrate consumption was found to provide positive results in avoiding and/or reducing decreases in performance and physiological responses in the second half [9]. However, so far, no studies have tested this combination. With the available findings, we may conclude that for an effective re-warm-up strategy, athletes should at least use some sort of external heat-retaining garments or perform an active routine $5-8 \mathrm{~min}$ before subsequent activity.

\subsection{Developed Warm-Up Programs}

As a result of the importance of a warm-up, some researchers have attempted to develop guidelines for warm-up protocols. First, Jeffreys [18] developed general recommendations with the "RAMP" method, which focused on three phases that should be of great importance for a good warm-up in all types of activities: (1) raise, which aims to elevate body temperature, heart rate, respiration rate, blood flow, and joint fluid viscosity via low-intensity activities; (2) activate and mobilize, which involves a series of specific dynamic key movement patterns involved in sports, together with a focus on key muscles that need to be activated to produce these movements; and (3) potentiation, which aims to increase activity to maximal intensity and potentiate PAP [18]. Specifically, in team sports, the FIFA Medical and Research Centre developed the FIFA 11+ warm-up program for football players and a recently injury prevention program for children called FIFA 11+ Kids [69]. The FIFA 11+ consists of running, strength, plyometric, and balance exercises [70] combining cardiovascular activation and neuromuscular exercise to reduce and prevent injury incidences in football players (for details, please see Soligard et al. [70]). Conversely, the FIFA 11+ Kids focuses on injury prevention and the development of special orientation, body stability, movement coordination, and fall techniques [71]. This study observed that application of this prevention warm-up program improved performance in dynamic balance, agility, jumping, and slalom dribbling (for details, see Rössler et al. [71]). Another program (Harmoknee) has been developed to prevent injuries in football players [72]. This program was developed to provide a warm-up that improved motion patterns and caused less strain to the knee joint [73]. It is recommended as a multi-faceted football-specific prevention program that combines education, proper motion patterns, strength, and balance, without using special equipment. Research indicates that this protocol resulted in a $77 \%$ 
decrease in the incidence of knee injury (for details, see Kiani et al. [72]).

Studies on these programs reported that FIFA $11+$ can be potentially effective for reducing the risk of injury and can enhance subsequent performance [73]. This program improved jump height, agility, and football skills while Harmoknee only improved football skills [74]. Although these programs have been proposed, only a few studies have evaluated their effects on athlete performance. Additionally, they are mainly directed towards football players and there is limited research on use of these programs in other sports [75]. Further research is needed to indicate possible adaptations that can be replicated for other team sports.

\subsection{Future Research}

Many warm-up protocols have been tested, with several identifying positive performance results. However, comparing these positive results to identify the best strategy is difficult owing to differences in the control groups. Future studies should have a common control group, with a structured strategy for comparison with other studies, aiming to identify the best methodology that results in the best player performance. Furthermore, there are many opportunities for bias that should be eliminated, as in the case of blinding and allocation concealment, to reduce the potential for bias to affect the results. Warm-up effects are usually assessed by sprints and jumps, but agility tests are valid and should be used also for analysis. In addition, new technology may allow further ecological analysis than what has been possible to date. The use of tracking systems such as a global positioning system in a real-world context could contribute to increased knowledge about warm-ups in team sports. This would facilitate the conduct of blinded studies, perhaps in the context of 'friendly' games and other settings, with the aim of gathering more data in ecological contexts for establishing external validity.

Given that in these types of competitions not all the athletes take the field at the same time, and that each player performs different functions, it seems important to know what specific warm-up components are best for the players' actions on the field. The possibility of applying specific warm-up strategies that differ between athletes should be investigated. It must be remembered that recommendations provided were based on a mean change and a small minority of individuals can respond differently. In addition, more research should be conducted on transition times, perhaps on combinations of active and passive strategies, as well as different interval times. Such studies would be of great importance for developing re-warm-up strategies and optimizing performance, especially in team sports. Additionally, most of the studies included focused on male athletes, and future investigations should also consider differences between sexes, ages, and training experiences and levels. The effect of time of the season should be analyzed, and warm-up data should be provided according to each phase of the annual training plan. It would also be interesting to understand the psychological aspects of a warm-up (for example, the belief of being ready after a warm-up), rarely investigated in team sports.

Future studies should aim to improve our knowledge of warm-up effects, by assessing and establishing different relationships between physical performance and physiological and biomechanical variables, which will increase the accuracy of feedback provided to optimize physical performance. By understanding the relationships between these variables, it could be possible to establish a comfortable range of the responses of physiological variables that are desirable immediately before the main physical task performance. Integrating this knowledge with recent technological innovations, for example, wearables, could provide a great opportunity to individualize each warm-up, leading to specific individual actions and increased efficiency of a warm-up and/or re-warm-up.

\subsection{Limitations}

Most of the results reported in the current review were based on the findings of single studies, limiting their applicability to alternative team sports and different populations. We found some limitations in the comparison of the results presented by different investigations, and specific recommendations concerning 'optimal' warm-up, post-warm-up, and re-warm-up strategies and exercise content were formulated based on the present set of data. Within the included studies, many strategies were investigated; however, they differed widely in the applied control group. Thus, it proved unfeasible to perform a meta-analysis, extrapolate conclusions, and compare strategies in various sports and populations. Furthermore, there were several methodological variations related to warm-up intensity, duration, recovery time, and type. These might partly account for the fact that warm-up protocols often vary significantly among players and sports.

\section{Conclusion}

The results of the critical analysis of the scientific literature performed in this review could translate into positive effects on short-term explosive performance. In team sports such as football, basketball, or rugby, which require athletes to sprint intermittently throughout the match [19], it is imperative that the warm-up improves acute explosive performance. To this end, it seems clear that the structure currently used for warm-ups should change. Implementing 
other strategies appears to be more effective in increasing explosive performance. The vast majority of studies on warm-ups obtained better results with a shorter and intensive warm-up. In general, the warm-up should be between 10 and 15 min, progressive in intensity $\left(\sim 50-90 \% \mathrm{HR}_{\max }\right)$, aim to increase body temperature and prepare for specific tasks of the sport, and end with tasks of maximum intensity such as sprints $\left(\sim 90 \% \mathrm{HR}_{\max }\right)$ to induce the PAP effect. Additionally, the warm-up benefits could be lost during transition phases between the warm-up and the start of a match if an effective post-warm-up strategy is lacking. This should be considered a critical component of the warm-up strategy. Although research indicates that the athlete should avoid sitting on the bench and would be better off standing, unfortunately this option is not possible owing to the rules of team sports. Studies suggest that it is essential, in light of these restrictions, to put on heated garments soon after a warm-up, to maintain muscle temperature. However, for transitions longer than $15 \mathrm{~min}$, a 2-min active re-warm-up with explosive tasks $\left(\sim 90 \% \mathrm{HR}_{\max }\right)$, just before entering the game, appears essential.

Regarding the half-time re-warm-up, it has been shown that rest should be avoided or minimized, as it causes a marked decrease in performance. In the current review, it was found that it was important to wear heated garments, to maintain muscle temperature, and to perform an active re-warm-up of 5 min with explosive tasks or small-sided games. Implementation of these strategies gives us an idea of whether an athlete is conditioned to perform well in sporting activities, but it remains unknown whether these isolated activities result in better sport performance on the field. Finally, we should emphasize that our findings were based on the results of studies conducted in commonly observed ambient conditions $\left(10-30{ }^{\circ} \mathrm{C}\right)$, and that studies conducted in extremely hot or cold conditions might have produced different results.

\section{Compliance with Ethical Standards}

Funding This project was supported by the Portuguese Foundation for Science and Technology and the European Union (UID/ DTP/04045/2013; POCI-01-0145-FEDER-006969) and also by the NanoSTIMA project, Macro-to-Nano Human Sensing: Towards Integrated Multimodal Health Monitoring and Analytics (NORTE-010145-FEDER-000016).

Conflict of interest Luís M. Silva, Henrique P. Neiva, Mário C. Marques, Mikel Izquierdo, and Daniel A. Marinho have no conflicts of interest directly relevant to the content of this review.

\section{References}

1. McCrary JM, Ackermann BJ, Halaki M. A systematic review of the effects of upper body warm-up on performance and injury. $\mathrm{Br}$ J Sports Med. 2016;49:935-42.

2. Bishop D. Warm up I: potential mechanisms and the effects of passive warm up on exercise performance. Sports Med. 2003;33(6):439-54.

3. Fradkin AJ, Zazryn TR, Smoliga JM. Effects of warming-up on physical performance: a systematic review with meta-analysis. J Strength Cond Res. 2010;24(1):140-8.

4. McGowan CJ, Pyne DB, Thompson KG, et al. Warm-up strategies for sport and exercise: mechanisms and applications. Sports Med. 2015;45:1523-46.

5. Neiva HP, Marques MC, Barbosa TM, et al. Warm-up and performance in competitive swimming. Sports Med. 2014;44:319-30.

6. Kilduff LP, Finn CV, Baker JS, et al. Preconditioning strategies to enhance physical performance on the day of competition. Int $\mathbf{J}$ Sports Physiol Perform. 2013;8(6):677-81.

7. Yiannis M. Performance and half time re-warm-up in soccer. Intl J Sport Std. 2014;4(11):1317-21.

8. Russell M, West DJ, Harper LD, et al. Half-time strategies to enhance second-half performance in team-sports players: a review and recommendations. Sports Med. 2014;45(3):353-64.

9. Racinais S, Oksa J. Temperature and neuromuscular function. Scand J Med Sci Sports. 2010;20(3):1-18.

10. Faulkner SH, Ferguson RA, Hodder SG, et al. External muscle heating during warm-up does not provide added performance benefit above external heating in the recovery period alone. Eur J Appl Physiol. 2013;113:2713-21.

11. Raccuglia M, Lloyd A, Filingeri D, et al. Post-warm-up muscle temperature maintenance: blood flow contribution and external heating optimisation. Eur J Appl Physiol. 2016;116:395-404.

12. Bishop D. Warm up II: performance changes following active warm up and how to structure the warm up. Sports Med. 2003;33(7):483-98.

13. Neiva HP, Morouço PG, Pereira FM, et al. O efeito do aquecimento no rendimento dos $50 \mathrm{~m}$ de nado. Motricidade. 2012;8(1):13-8.

14. Neiva HP, Marques MC, Fernandes RJ, et al. Does warm-up have a beneficial effect on 100-m freestyle? Int J Sports Physiol Perform. 2014;9:145-50.

15. Ayala F, Moreno-Pérez V, Vera-Garcia FJ, et al. Acute and time-course effects of traditional and dynamic warmup routines in young elite junior tennis players. PLoS One. 2016;11(4):e0152790.

16. Zois J, Bishop D, Aughey R. High-intensity warm-ups: effects during subsequent intermittent exercise. Int J Sports Physiol Perform. 2015;10:498-503.

17. McMillian DJ, Moore JH, Hatler BS, et al. Dynamic vs. staticstretching warm up: the effect on power and agility performance. J Strength Cond Res. 2006;20:492-9.

18. Jeffreys I. Warm up revisited: the ramp method of optimising performance preparation. Prof Strength Cond. 2007;6:12-8.

19. Edge J, Bishop D, Goodman C, et al. Effects of high and moderate-intensity training on metabolism and repeated sprints. Med Sci Sports Exerc. 2005;37(11):1975-82.

20. Marques MC, Izquierdo M. Kinetic and kinematic associations between vertical jump performance and $10-\mathrm{m}$ sprint time. J Strength Cond Res. 2014;28(8):2366-71.

21. Bishop D, Girard O, Villanueva A. Repeated-sprint ability. Part II: recommendations for training. Sports Med. 2011;41(9):741-56.

22. Girard O, Villanueva A, Bishop D. Repeated-sprint ability. Part I: factors contributing to fatigue. Sports Med. 2011;41(8):673-94. 
23. Moher D, Shamseer L, Clarke M, et al. Preferred reporting items for systematic review and meta-analysis protocols (PRISMA-P) 2015 statement. Syst Rev. 2015;1(4):1.

24. Methley AM, Campbell S, Chew-Graham C, et al. PICO, PICOS and SPIDER: a comparison study of specificity and sensitivity in three search tools for qualitative systematic reviews. BMC Health Serv Res. 2014;14:579.

25. Higgins JP, Green S. Cochrane handbook for systematic reviews of interventions, version 5.1.0 [updated March 2011]. The Cochrane Collaboration, editors. 2011. http://www.handbook.cochrane.org. Accessed 28 Nov 2017.

26. Conboy JE. Algumas medidas típicas univariadas da magnitude do efeito. Análise Psicológica. 2003;2(XXI):145-58.

27. Cohen J. Statistical power analysis for the behavioural sciences. Hillsdale: Erlbaum; 1988.

28. Abade E, Sampaio J, Gonçalves B, et al. Effects of different rewarm-up activities in football players' performance. PLoS One. 2017;12(6):1-13.

29. Morton NA. The PEDro scale is a valid measure of the methodological quality of clinical trials: a demographic study. Aust J Physiother. 2009;55:129-33.

30. Ayala F, Baranda PS, Croix MD. Stretching in warm-up: design of routines and their impact on athletic performance. Rev Int Med Cienc Act Fís Deporte. 2012;12(46):349-68.

31. Andrade DC, Henriquez-Olguín C, Beltrán AR, et al. Effects of general, specific and combined warm-up on explosive muscular performance. Biol Sport. 2015;32(2):123-8.

32. Tomaras EK, MacIntosh BR. Less is more: standard warm-up causes fatigue and less warm-up permits greater cycling power output. J Appl Physiol. 2011;111(1):228-35.

33. Mujika I, de Txabarri RG, Maldonado-Martín S, et al. Warmup intensity and duration's effect on traditional rowing time-trial performance. Int J Sports Physiol Perform. 2012;7(2):186-8.

34. Zois J, Bishop DJ, Ball K, Aughey RJ. High-intensity warm-ups elicit superior performance to a current soccer warm-up routine. J Sci Med Sport. 2011;14(6):522-8.

35. Sue RA, Adams KJ, DeBeliso M. Optimal timing for post-activation potentiation in women collegiate volleyball players. Sport J. 2016;4(27):1-9.

36. Taylor JM, Weston M, Portas MD. The effect of a short, practical warm-up protocol on repeated-sprint performance. J Strength Cond Res. 2013;27(7):2034-8.

37. Tillaar R, Heimburg E. Comparison of two types of warm-up upon repeated sprint performance in experienced soccer players. J Strength Cond Res. 2016;30(8):2258-65.

38. Pardeiro M, Yanci J. Warm-up effects on physical performance and psychological perception in semi professional soccer players. Rev Int Cienc Deporte. 2017;13(48):104-16.

39. Yaicharoen P, Wallman K, Morton A, et al. The effect of warm-up on intermittent sprint performance and selected thermoregulatory parameters. J Sci Med Sport. 2012;15(5):451-6.

40. Meckel Y, Gottieb R, Eliakem A. Repeated sprint tests in young basketball players at different game stages. Eur J Appl Physiol. 2009;107(3):273-9.

41. Anderson P, Landers G, Wallman K. Effect of warm-up on intermittent sprint performance. Res Sports Med. 2014;22(1):88-99.

42. Guinoubi C, Sahli H, Mekni R, et al. Effects of two warm-up modalities on short-term maximal performance in soccer players: didactic modeling. Adv Health Sci Educ Theory Pract. 2015;5:70-6.

43. Tillaar R, Lerberg E, Heimburg E. Comparison of three types of warm-up upon sprint ability in experienced soccer players. J Sport Health Sci. 2016. https://doi.org/10.1016/j.jshs.2016.05.006.

44. Turki O, Chaouachi A, Drinkwater EJ, et al. Ten minutes of dynamic stretching is sufficient to potentiate vertical jump performance characteristics. J Strength Cond Res. 2011;25(9):2453-63.
45. Cilli M, Gelen E, Yildiz S, et al. Acute effects of a resisted dynamic warm-up protocol on jumping performance. Biol Sport. 2014;31(4):277-82.

46. Pojskic H, Pagaduan J, Uzicanin E, et al. Acute effects of loaded whole body vibration training on performance. Asian J Sports Med. 2015;6(1):1-7.

47. Gabbett TJ, Sheppard JM, Pritchard-peschek KR, et al. Influence of closed skill and open skill warm-ups on the performance of speed, change of direction speed, vertical jump, and reactive agility in team sport athletes. J Strength Cond Res. 2013;2(5):1413-5.

48. Marinho DA, Gil MH, Marques MC, et al. Complementing warmup with stretching routines: effects in sprint performance. Sports Med Int Open. 2017;1:101-6.

49. Pojskić H, Pagaduan JC, Babajić F, et al. Acute effects of prolonged intermittent low-intensity isometric warm-up schemes on jump, sprint, and agility performance in collegiate soccer players. Biol Sport. 2015;32(2):129-34.

50. Jamshidi M, Jahromi MK, Salesi M, et al. The effect of three methods of warm-up on the anaerobic power, agility, speed, flexibility and fatigue index of elite female volleyball players. Turk J Kinesiol. 2016;2(3):34-42.

51. Turki O, Chaouachi A, Behm DG, et al. The effect of warm-ups incorporating different volumes of dynamic stretching on 10and $20-\mathrm{m}$ sprint performance in highly trained male athletes. $\mathrm{J}$ Strength Cond Res. 2012;26(1):63-72.

52. Beckett JR, Schneiker KT, Wallman KE, et al. Effects of static stretching on repeated sprint and change of direction performance. Med Sci Sports Exerc. 2009;41(2):444-50.

53. McHugh MP, Cosgrave $\mathrm{CH}$. To stretch or not to stretch: the role of stretching in injury prevention and performance. Scand J Med Sci Sports. 2010;20(2):169-81.

54. Behm DG, Chaouachi A. A review of the acute effects of static and dynamic stretching on performance. Eur J Appl Physiol. 2011;111(11):2633-51.

55. Carvalho FL, Carvalho MC, Simão R, et al. Acute effects of a warm-up including active, passive, and dynamic stretching on vertical jump performance. J Strength Cond Res. 2012;26(9):2447-52.

56. Peck E, Chomko G, Gaz D, et al. The effects of stretching on performance. Curr Sports Med Rep. 2014;13(3):179-85.

57. Sampaio-Jorge F, Rangel LF, Mota HR, et al. Acute effects of passive stretching on muscle power performance. J Exerc Physiol Online. 2014;17(6):81-9.

58. Little T, Williams AG. Effects of differential stretching protocols during warm-ups on high-speed motor capacities in professional soccer players. J Strength Cond Res. 2006;20(1):203-307.

59. Crowther RG, Leicht AS, Pohlmann JM, et al. Influence of rest on players' performance and physiological responses during basketball play. Sports. 2017;5(27):1-6.

60. Galazoulas C, Tzimou A, Karamousalidis G, et al. Gradual decline in performance and changes in biochemical parameters of basketball players while resting after warm-up. Eur J Appl Physiol. 2012;112(9):3327-34.

61. Alberti G, Annoni M, Ongaro L, et al. Athletic performance decreases in young basketball players after sitting. Int J Sports Sci Coach. 2014;9(5):975-84.

62. West DJ, Russell M, Bracken RM, et al. Post-warmup strategies to maintain body temperature and physical performance in professional rugby union players. J Sports Sci. 2016;34(2):110-5.

63. Kilduff LP, West DJ, Williams N, et al. The influence of passive heat maintenance on lower body power output and repeated sprint performance in professional rugby league players. J Sci Med Sport. 2013;5:482-6.

64. Mohr M, Mujika I, Santisteban J, et al. Examination of fatigue development in elite soccer in a hot environment: a multi-experimental approach. Scand J Med Sci Sports. 2010;3:125-32. 
65. Mohr M, Krustrup P, Nybo L, et al. Muscle temperature and sprint performance during soccer matches: beneficial effect of re-warm-up at half-time. Scand J Med Sci Sports. 2004;14(3):156-62.

66. Zois J, Bishop D, Fairweather I, et al. High-intensity re-warm-ups enhance soccer performance. Int J Sports Med. 2013;34(9):800-5.

67. Edholm P, Krustrup P, Randers MB. Half-time re-warm up increases performance capacity in male elite soccer players. Scand J Med Sci Sports. 2015;25:40-9.

68. Russell M, West DJ, Briggs MA, et al. A passive heat maintenance strategy implemented during a simulated half-time improves lower body power output and repeated sprint ability in professional rugby union players. PLoS One. 2015;10(3):e0119374.

69. Rössler R, Junge A, Bizzini M, Verhagen E, et al. A multinational cluster randomised controlled trial to assess the efficacy of ' $11+$ Kids': a warm-up programme to prevent injuries in children's football. Sports Med. 2018;48(6):1493-504.

70. Soligard T, Myklebust G, Steffen K, et al. Comprehensive warmup programme to prevent injuries in young female footballers: cluster randomised controlled trial. BMJ. 2008;337:1-9.

71. Rössler R, Donath L, Bizzini M, et al. A new injury prevention programme for children's football, FIFA 11+ Kids, can improve motor performance: a cluster-randomised controlled trial. J Sports Sci. 2016;34(6):549-56.

72. Kiani A, Hellquist E, Ahlqvist K, et al. Prevention of soccer-related knee injuries in teenaged girls. Arch Intern Med. 2010;170(1):43-9.
73. Bizzini M, Impellizzeri FM, Dvorak J, et al. Physiological and performance responses to the "FIFA 11+" (part 1): is it an appropriate warm-up? J Sports Sci. 2013;31(13):1481-90.

74. Daneshjoo A, Mokhtar AH, Rahnama N, et al. Effects of the 11+ and Harmoknee warm-up programs on physical performance measures in professional soccer players. J Sports Sci Med. 2013;12(3):489-96.

75. Longo UG, Loppini M, Berton A, et al. The FIFA 11+ program is effective in preventing injuries in elite male basketball players: a cluster randomized controlled trial. Am J Sports Med. 2012;40(5):996-1005.

76. Barry L, Kenny I, Comyns T. Performance effects of repetition specific gluteal activation protocols on acceleration in male rugby union players. J Hum Kinet. 2016;54:33-42.

77. Lovell R, Midgley A, Barrett S, et al. Effects of different halftime strategies on second half soccer-specific speed, power and dynamic strength. Scand J Med Sci Sports. 2013;23(1):105-13.

78. Okuno NM, Tricoli V, Silva SB, et al. Postactivation potentiation on repeated-sprint ability in elite handball players. J Strength Cond Res. 2013;27(3):662-8.

79. Rønnestad BR, Ellefsen S. The effects of adding different whole body vibration frequencies to preconditioning exercise on subsequent sprint performance. J Strength Cond Res. 2011;25(12):3306-10.

80. Sander A, Keiner M, Schlumberger A, et al. Effects of functional exercises in the warm-up on sprint performances. J Strength Cond Res. 2013;27(4):995-1001.

\section{Affiliations}

\section{Luís Miguel Silva ${ }^{1} \cdot$ Henrique Pereira Neiva ${ }^{1,2} \cdot$ Mário Cardoso Marques $^{1,2} \cdot$ Mikel Izquierdo $^{3}$ (1) Daniel Almeida Marinho ${ }^{1,2}$}

Luís Miguel Silva

luis.silva@ubi.pt

Henrique Pereira Neiva

hpn@ubi.pt

Mário Cardoso Marques

mmarques@ubi.pt

Daniel Almeida Marinho

dmarinho@ubi.pt
1 Department of Sport Sciences, University of Beira Interior, Covilhã, Portugal

2 Research Centre in Sport Sciences, Health Sciences and Human Development (CIDESD), Covilhã, Portugal

3 Department of Health Sciences, Public University of Navarre, Tudela, Spain 